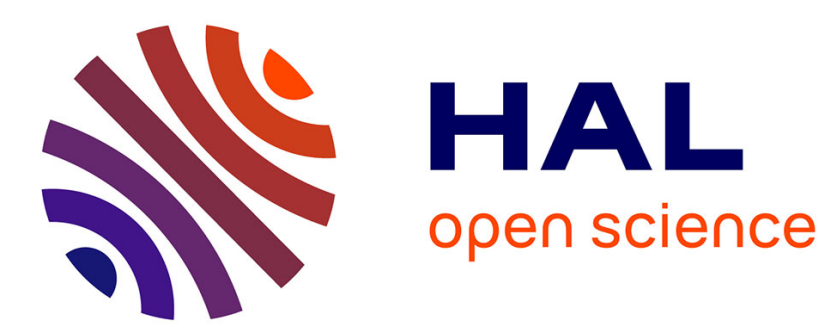

\title{
La mobilité comme variabilité temporelle de la présence spatiale \\ Hadrien Commenges
}

\section{To cite this version:}

Hadrien Commenges. La mobilité comme variabilité temporelle de la présence spatiale. Flux - Cahiers scientifiques internationaux Réseaux et territoires, 2014, 1 (95), pp.41-55. halshs-01021581

\section{HAL Id: halshs-01021581 https://shs.hal.science/halshs-01021581}

Submitted on 9 Jul 2014

HAL is a multi-disciplinary open access archive for the deposit and dissemination of scientific research documents, whether they are published or not. The documents may come from teaching and research institutions in France or abroad, or from public or private research centers.
L'archive ouverte pluridisciplinaire HAL, est destinée au dépôt et à la diffusion de documents scientifiques de niveau recherche, publiés ou non, émanant des établissements d'enseignement et de recherche français ou étrangers, des laboratoires publics ou privés. 
Publié dans la revue Flux, 2014/1, no95, pp.41-55

Site de la revue : http://olegk.free.fr

Accès sur Cairn : http://www.cairn.info/revue-flux-2014-1.htm

\title{
La mobilité comme variabilité temporelle de la présence spatiale
}

\author{
Hadrien Commenges \\ Université Paris Diderot - Paris 7 \\ UMR 8504 Géographie-cité
}

\section{Introduction}

Les premières mesures systématiques des déplacements apparaissent en France en 1844 sous le nom de « recensement de la circulation». Ces recensements servent à quantifier la circulation sur les routes nationales, «opération envisagée au double point de vue de la statistique commerciale des transports par voie de terre et de l'usure des chaussées » (Ministère des Travaux Publics, 1918, p. 9). À la fin des années 1950 un nouveau type de dispositif de mesure est importé des États-Unis par des ingénieurs des Ponts et Chaussées envoyés dans les universités américaines (Dupuy, 1975) : l'enquête origine-destination. Il existe une certaine variété d'enquêtes de ce type (enquêtes ménages, enquêtes cordons, etc.), mais toutes produisent le même type d'objets : des couples origine-destination. Les enquêtes origine-destination réalisées au domicile des ménages, ou «enquêtes ménages déplacements » (1), font l'objet de l'action normalisatrice des services de l'État dès le milieu des années 1970 (SETRA, 1975) et font preuve d'une grande stabilité jusqu'à aujourd'hui.

L'enquête ménages déplacements ne subit que des modifications marginales malgré plusieurs évolutions contextuelles majeures. D'abord, les progrès de l'informatique changent la donne en termes d'acquisition, d'analyse et de représentation des déplacements. Ensuite, de nouveaux dispositifs apparaissent permettant une géolocalisation des individus en temps réel. Enfin, les approches de la mobilité des individus et les questions susceptibles d'être posées ont évolué depuis les années 1950. Ces évolutions affectent peu le modèle conceptuel des données d'enquêtes ménages déplacements (EMD), conçu comme l'ensemble des objets produits par l'enquête, leur nature, leurs interrelations et leurs attributs.

La thèse soutenue dans cet article est que le modèle conceptuel de données constitue bien plus qu'un simple formatage des données, il définit la matière de l'analyse et façonne les questionnements, les traitements de données et les interprétations des résultats. Comme le spectateur devant un tableau dont «l'œil suit les chemins qui lui ont été ménagés dans l'œuvre»(Paul Klee, cité par Perec, 1978), l'utilisateur d'une telle base de données dispose d'une liberté canalisée par le modèle conceptuel des données qu'il analyse.

Les travaux retraçant l'histoire des instruments destinés à la planification des transports (Dupuy, 1975 ; Facq, 2006 ; Chatzis, 2009, Commenges 2013) amènent à considérer que le dispositif d'enquête et son modèle conceptuel de données sont issus d'un contexte technico-scientifique très particulier, celui de l'ingénierie du trafic (traffic engineering). Dans ce cadre, le transport est considéré comme un bien immatériel dont la demande est exprimée en termes de déplacements. Le déplacement, mouvement réalisé sur la voie publique reliant deux motifs (CERTU, 1998), est la clef de voûte de l'armature conceptuelle et méthodologique de l'ingénierie et de l'économie des transports. Le dispositif d'enquête, l'EMD, est littéralement un dispositif qui recueille les déplacements réalisés par les ménages. Depuis les premières enquêtes origine-destination réalisées aux États-Unis dans les années 1940 (Weiner, 1997), de nouvelles approches ont été proposées s'intéressant aux individus et à leurs activités, assorties de nouveaux types d'enquêtes. Cependant, l'EMD est restée le dispositif dominant de mesure de la mobilité jusqu'à aujourd'hui, et elle porte toujours le modèle conceptuel de données originel, qualifié ici de modèle déplacement-centré. 
Cet article décortique ce modèle de données pour mettre à jour la façon dont il guide les questionnements, les traitements et les interprétations vers une approche déplacement-centrée. La démarche est conduite en trois temps: une analyse détaillée du modèle conceptuel historique des EMD; une proposition de transformation de ce modèle de données vers un modèle qualifié de séquentiel, qui s'appuie sur des travaux anciens issus de la time-geography et sur des travaux plus récents sur les patrons d'activités; et enfin un exemple qui compare les résultats de deux analyses thématiquement comparables menées en parallèle dans une approche classique déplacement-centrée et dans une approche séquentielle. Cette comparaison met en avant la complémentarité des deux approches : concevoir la mobilité comme mouvement dans l'espace et la concevoir comme variabilité temporelle de la présence spatiale.

\section{Le modèle conceptuel historique des EMD}

Le terme de modèle conceptuel de données (MCD) revêt un sens très précis dans les sciences informatiques. Les formalismes utilisés dans ce domaine (MERISE, UML) sont à la fois stricts, assez récents et orientés vers une implémentation informatique. Les organismes tels que l'Insee ou le CERTU utilisent, pour décrire les données qu'ils produisent, les termes de «dessin de fichiers» et de « dictionnaire de variables». En effet, les relations conceptuelles entre les différents objets statistiques produits par une enquête (le ménage, l'individu, etc.) sont assez peu mises en avant.

Cependant, tout ensemble d'objets statistiques dotés d'attributs et reliés techniquement (par un identifiant) et conceptuellement les uns aux autres peut être considéré comme un MCD, même si ce dernier est peu formalisé. Cette définition plus souple, qui ne s'inscrit pas dans les sciences informatiques, permet de remonter avant les années 1970, qui marquent le début des modèles conceptuels au sens strict, pour s'intéresser à tous les travaux visant à décrire le produit d'un dispositif d'enquête quantitative, en particulier ceux de P. F. Lazarsfeld et H. Menzel (1961). Le modèle conceptuel est donc défini ici comme l'ensemble des objets produits par une enquête quantitative, leur nature, leurs interrelations et leurs attributs.

\section{Aspects techniques et cognitifs de la mise en forme de l'information}

La conception et la réalisation d'une enquête, sa mise en forme numérique et les exploitations de l'enquête forment un trio dont les liens doivent être précisés. Le processus de construction de l'information quantitative fait l'objet de travaux dans de nombreux domaines: les catégories socio-professionnelles (Desrosières et Thévenot, 1979), les catégories ethniques (Schor, 2001), la statistique agricole (Didier, 2009), les chiffres du chômage (Salais et alii, 1986). Tous ces travaux ont en commun de considérer que les dispositifs statistiques ne sont pas neutres ni transparents, qu'ils ne sont pas de simples leviers au service de l'action publique, mais qu'ils contribuent à structurer le contenu de cette action et à en façonner les contours, en somme ils ont des « effets spécifiques » (Ihl et alii, 2003 ; Lascoumes et Le Galès, 2004).

Au-delà de ce constat, il convient de dégager les mécanismes qui confèrent aux instruments de mesure ces « effets spécifiques ». En première analyse, la conception de l'enquête et sa mise en forme dans un modèle de données répondent aux besoins des exploitations primaires de l'enquête. En ce sens ces deux produits, enquête et MCD, sont soumis à l'exploitation et à l'interprétation et ils n'ont donc pas d'effets spécifiques. Les exploitations secondaires de l'enquête doivent faire avec : l'enquête mise en forme dans un MCD rend certaines exploitations secondaires impossibles quand l'information est absente, ou difficiles quand le MCD doit subir des transformations massives.

Au-delà de ces deux cas, impossibilité et difficulté technique, un troisième cas se dégage, celui de la difficulté conceptuelle. Le MCD n'est pas une simple mise en forme de l'information, c'est aussi une mise en forme conceptuelle: les objets statistiques accompagnés de leurs propriétés sont des portions du phénomène à l'étude que le MCD fait exister en les réifiant. Le codage statistique revêt donc un aspect technique mais il revêt également un aspect cognitif (Desrosières, 1989). Ainsi, la mise en forme de l'enquête peut rendre les exploitations secondaires difficiles à réaliser mais également difficiles à penser. 
Le travail présenté par la suite correspond précisément au cas où une utilisation alternative est possible mais rendue techniquement et conceptuellement difficile. Il s'agit d'appliquer cette idée aux enquêtes ménages déplacements pour analyser de quelle façon leur mise en forme dans un MCD guide leur exploitation.

\section{La mise en forme de la mobilité quotidienne par les EMD}

Les EMD se développent aux États-Unis à partir des années 1940 et sont importées en Europe occidentale à la fin des années 1950 (Dupuy, 1975 ; Weiner, 1997). Les enquêtes des grandes métropoles américaines et européennes sont comparables quant aux objets qu'elles produisent et quant aux attributs de ces objets. Elles sont constituées d'un ensemble de quatre objets : des ménages, des individus, des déplacements et des moyens ou étapes. Chacun de ces quatre objets est défini sur un critère d'unité. La définition de l'objet ménage sur l'unité de logement est celle de l'Insee et n'est pas propre aux EMD. L'objet individu contient un critère d'unité puisqu'il est intrinsèquement et étymologiquement indivisible. L'objet déplacement se définit par l'unité de motif, c'est un mouvement réalisé sur la voie publique reliant deux motifs (CERTU, 1998), un lien entre deux activités. L'attribut «motif» est la base sur laquelle le continuum mobile est découpé en une série discrète de déplacements. L'objet étape se définit par l'unité de mode : une étape est une partie du déplacement réalisée avec un même mode.

Les liens inter-objets sont de deux types : le lien central est le lien de réalisation, des individus réalisent des déplacements / des déplacements sont réalisés par des individus. Les deux autres objets, étape et ménage, servent à caractériser plus finement chacun des deux objets fondamentaux que sont l'individu et le déplacement, ils sont reliés à eux par des liens de composition. Les ménages sont composés d'individus, les déplacements sont composés d'étapes. L'objet étape n'existe pas dans les premières enquêtes de mobilité (SERC, 1964 ; SERC, BRC de Rennes, 1964) et n'est défini qu'à la fin des années 1970. L'objet ménage existe dès les origines, parce qu'il constitue la base d'échantillonnage et parce que certaines de ses propriétés sont jugées indispensables dans les modèles de prévision.

Le produit de l'enquête prend la forme d'une base de quatre tableaux (Ménage, Individu, Déplacement, Étape) reliés entre eux par un identifiant. Cet ensemble de quatre objets accompagnés de leurs attributs forme le modèle de données historique des EMD. Préciser le contenu du modèle conceptuel de données sur la mobilité consiste à définir la nature des objets qui le composent, à étudier leurs propriétés et à définir les relations entre ces objets. Les développements qui suivent visent à en préciser le contenu par une lecture des liens inter-objets. Ce sont ces liens qui marquent le cadre dans lequel la mobilité quotidienne est appréhendée, cadre qui canalise les questionnements et les exploitations.

\section{La prépondérance de l'objet déplacement}

L'objet déplacement occupe une place prépondérante dans l'analyse et la modélisation de la mobilité quotidienne. Cette section vise à acter ce fait, mais également à examiner en quoi il est le produit de l'interaction entre l'exploitation de l'enquête et sa mise en forme dans le MCD.

Le dispositif d'enquête ménages déplacements naît dans le cadre du développement de l'économie des transports pour alimenter les modèles de prévision et d'évaluation des choix d'investissements (SERC, 1964 ; Dupuy, 1975; Chatzis, 2009). Les modèles de prévision classiques estiment une demande quantifiée en nombre de déplacements, distribuent ces déplacements sur un espace par l'intermédiaire d'un modèle, le plus souvent gravitaire, puis assignent ces déplacements à des modes et à des itinéraires. Dans cette approche de modélisation, l'offre et la demande sont donc mesurées en termes de déplacements.

Cette finalité originelle du dispositif d'enquête amène une description des pratiques de mobilité axée sur le déplacement. Les rapports d'enquête décrivent précisément les déplacements en les caractérisant par un couple de variables absolues (en particulier le mode, le motif et l'horaire) et de variables contextuelles (2) se référant aux individus (sexe, âge, catégorie socio-professionnelle) : «La courbe de trafic [i.e. la 
répartition horaire des déplacements] des scolaires et étudiants est assez semblable à celles des actifs » (Dreyfus, 2005, p. 19). «Les déplacements professionnels sont encore très masculins: $54 \%$ des déplacements vers le travail et $71 \%$ de ceux pour activités professionnelles sont effectués par des hommes » (Courel et alii, 2005a, p. 29). Parallèlement, les rapports d'enquête décrivent les déplacements en les caractérisant par un couple de variables absolues (mode, motif, horaire) et de variables contextuelles se référant à l'espace, et plus particulièrement à un zonage spatial : «En 2001, 62\% des déplacements en TC sont liés à Paris et $38 \%$ sont internes à la banlieue, contre respectivement $69 \%$ et $31 \%$ en 1976 » (Courel et alii, 2005b, p. 1). «En 2001, seuls 30,1\% des déplacements en transports collectifs ont une extrémité en Grande Couronne » (Bernard et alii, 2005, p. 3).

Le déplacement devient ainsi le pivot de la description de la mobilité, les autres objets internes à l'enquête (individus et ménages) ou externes à l'enquête (zonage spatial) servant à préciser le contexte sociodémographique et le contexte spatial de ces déplacements. Cette approche déplacement-centrée est en premier lieu dictée par l'objectif opérationnel de la description et de la modélisation de la mobilité et par l'ancrage historique de cet objectif.

Cependant, il est aussi possible de considérer que la prépondérance de l'objet déplacement vient du fait que le modèle conceptuel de données guide l'exploitation de l'enquête vers des analyses déplacementcentrées. En effet, presque toute l'information relative à la mobilité est concentrée dans les propriétés de l'objet déplacement (3). Ce constat s'applique sans réserve à l'Enquête Globale Transport utilisée dans la troisième section de l'article. Dans le cas des EMD «standard CERTU », ce n'est qu'à partir des années 2000 qu'elles commencent à intégrer des informations sur la mobilité au niveau de l'individu, en particulier sur la fréquence d'usage des différents modes de transport ou sur l'image que se font les individus de ces modes (pratique, confortable, dangereux, etc.). L'utilisateur de ces données qui s'intéresse principalement à l'individu et désire adopter une approche individu-centrée doit donc très souvent se contenter de propriétés dérivées de l'objet déplacement.

Ainsi, le modèle conceptuel de données de l'EMD et la description des déplacements sont relativement bien adaptés aux objectifs opérationnels de la prévision et de l'évaluation économique. L'exploitation primaire des données d'enquête conserve donc les traits qu'elle avait depuis les origines. Les autres champs disciplinaires se cantonnent à mettre au point d'autres dispositifs d'enquêtes ( $c f$. section suivante) ou bien à réaliser des exploitations secondaires des EMD, raison pour laquelle ils n'auront aucun impact sur la redéfinition du modèle de données.

\section{Un modèle de données pour décrire les pratiques spatiales des individus}

À la fin des années 1970, un courant de recherches se développe autour de l'analyse des modes de vie. Plusieurs auteurs mènent des études autour des comportements de mobilité en déplaçant le centre d'intérêt vers les individus et les ménages (Haumont et alii, 1977 ; Haumont et alii, 1978 ; Raux et alii, 1988). Cette approche s'inscrit dans un mouvement plus vaste qui prend ses racines dans le développement de la time-geography (Chapin, 1978). Ces recherches s'appuient sur des dispositifs d'enquête originaux (Raux et alii, 1988) qui s'écartent du modèle des EMD. Cependant, elles sont réalisées dans le cadre de projets de recherche spécifiques et ne s'imposent pas comme dispositifs pérennes. Les EMD conservent donc leur statut de dispositif dominant appuyé par les besoins opérationnels des administrations et des opérateurs de transport. Il reste cependant possible de faire avec, en transformant le modèle de données historique pour le destiner à une analyse des pratiques individuelles de mobilité dans le style des approches activitéscentrées.

\section{Les données de l'EMD comme données longitudinales}

Les EMD sont des enquêtes transversales (cross sectional survey), elles produisent un photo de l'état de la mobilité à un moment donné. Ces enquêtes comportent en général plusieurs vagues (CERTU, 1998) mais il n'y a pas de mesures répétées sur les mêmes individus et il ne s'agit donc pas d'un dispositif longitudinal (longitudinal research design). Il faut cependant distinguer le type de données produites et le 
type de dispositif d'enquête. Le dispositif d'enquête est qualifié de longitudinal lorsqu'il procède à des mesures répétées au cours du temps sur les mêmes individus statistiques. Les données sont qualifiées de longitudinales lorsqu'elles qualifient ces individus à différents moments. Ainsi une enquête à une seule vague (donc non longitudinale) peut parfaitement produire des données longitudinales (Taris, 2000). Il est possible de considérer que les EMD produisent une information longitudinale par des questions rétrospectives, du fait qu'elles interrogent les individus sur l'ensemble des déplacements réalisés la veille.

Le fait de considérer le produit de l'EMD comme des données longitudinales amène à leur appliquer des méthodes et des modèles conceptuels de données adaptés à ce changement de perspective, et plus particulièrement des méthodes d'analyse séquentielle (Abbott, 1995). Certains auteurs ont déjà appliqué ces méthodes à l'analyse des programmes d'activités. Les «actogrammes » (4) (cf. Figure 1) présentent ainsi des séquences d'activités et de déplacements réalisés tout au long de la journée (Bonnafous et alii, 1981 ; Joh et alii, 2002 ; Chardonnel et alii, 2004 ; Thévenin et alii, 2007).

L'exploitation de données séquentielles mobilise trois dimensions: (i) le temps discret constitué d'un ensemble de tranches temporelles, (ii) les séquences représentant les individus statistiques à l'étude, et (iii) l'alphabet représentant l'ensemble des états observés. Cette troisième dimension donne du contenu aux deux premières, qui sont les dimensions structurantes. Les deux dimensions, séquentielle et temporelle, amènent à proposer une double lecture des données : la lecture horizontale s'intéresse à la succession d'états au sein de chaque séquence. Elle est dite séquentielle parce qu'elle s'applique à une liste ordonnée d'états : elle s'intéresse à la fois à l'occurrence des états et à l'ordre de ces occurrences. Cette lecture répond aux deux questions formalisées par Abbott et Hysrcha (1990) : la pattern question (ressemblance et dissemblance entre les séquences) et la generation question (production des séquences). La lecture verticale s'intéresse à la distribution des états au sein de chaque tranche temporelle. Elle agrège les états des séquences et décrit la co-évolution des états au cours de l'axe temporel.

\section{Figure 1. Actogrammes d'un échantillon de 10 individus}

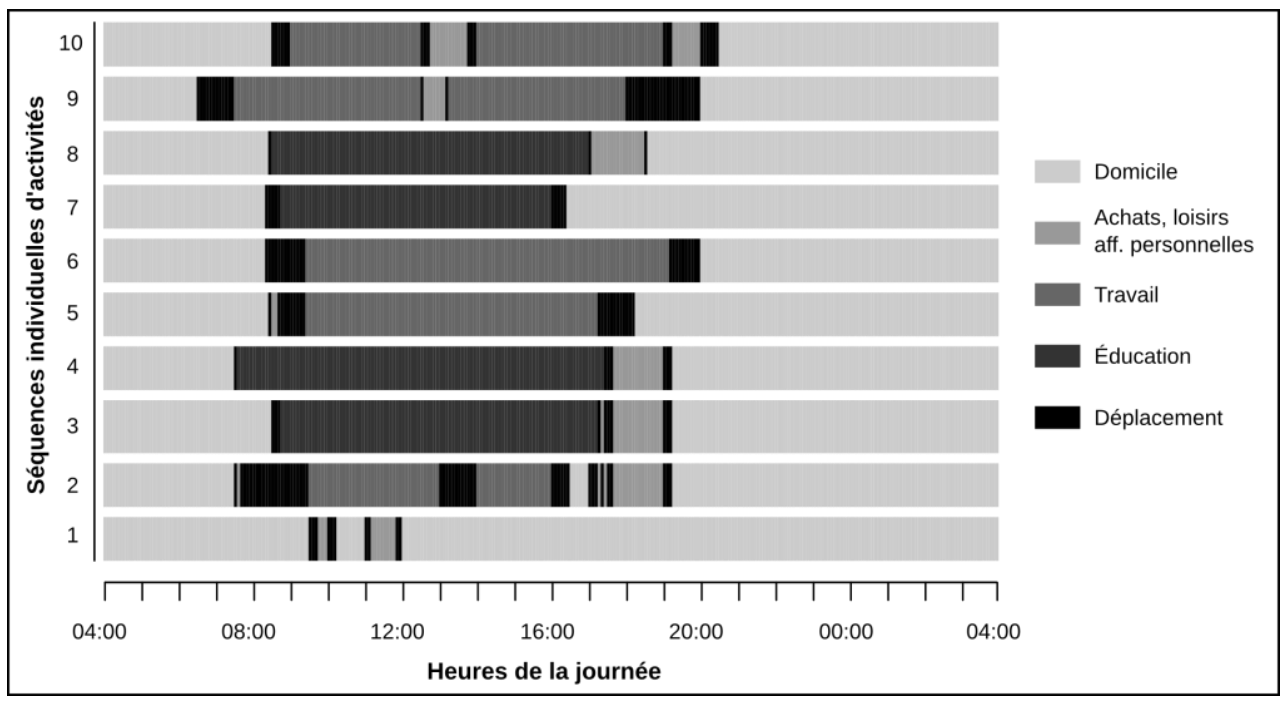

Source : Enquête Globale Transport 2001-2002 - Réalisation : H.Commenges

Un actogramme est un ensemble de séquences d'activités. Cette représentation des activités peut facilement être étendue à tout état caractérisant les individus en faisant remonter les propriétés des objets de niveau inférieur : les déplacements et les étapes. Avant de préciser les modalités de cette reconstruction, il convient de préciser les notions de séquence, d'état, d'événement et d'épisode ( $c f$. Figure 2). Une séquence est une liste ordonnée d'états ou d'événements découpée par une unité de temps discrète. Un état est une propriété de l'objet d'intérêt qui a une durée, alors qu'un événement est une propriété sans durée. Tout changement d'état est un événement, et tout événement marque un changement d'état (Gabadinho $e t$ 
alii, 2009). Un épisode est une succession de plusieurs états similaires. À l'instar de la définition de la notion d'activité par As (1978), l'état est défini de façon très générale comme n'importe quelle propriété caractérisant l'objet d'intérêt (ici l'individu) sur un segment temporel donné. Le principe général de la reconstruction du modèle de données est de réorganiser toute l'information contenue dans les objets déplacement et étape autour de l'objet individu, en les considérant comme des états. Cette démarche constitue plus qu'une simple manipulation préalable à une représentation graphique (l'actogramme), il s'agit d'une véritable refonte du modèle de données traditionnel en un modèle de données séquentiel.

Figure 2. Définition des notions d'état, d'évènement et d'épisode

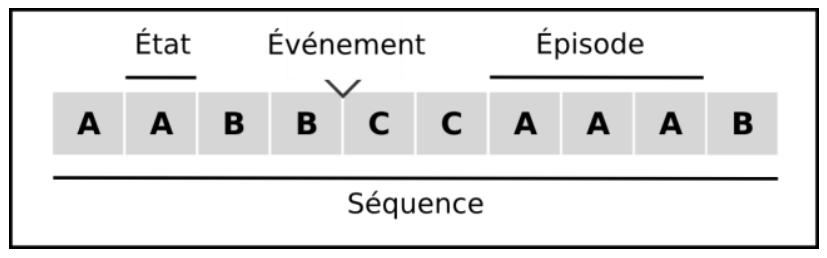

Source : Brzinsky-Fay et al. 2005 - Réalisation : H.Commenges

Cette refonte entraîne une simplification du modèle de données originel : il ne contient plus qu'un seul objet, l'individu, caractérisé par des propriétés contextuelles, des propriétés absolues et des propriétés temporelles ou états. Les propriétés temporelles sont des propriétés « estampillées » (timestamped), c'està-dire localisées dans le temps (Parent et alii, 1997). Cette localisation fait référence à un axe temporel qui ne peut être que celui hérité du modèle de données originel. Il s'agit d'un axe temporel de processus et non d'un axe temporel de calendrier (Blossfeld et Rohwer, 2002) : il débute avec le premier état de chaque individu, indépendamment du temps de calendrier. Dans le cas des EMD, l'axe temporel commence à quatre heures du matin et se termine à quatre heures du matin du jour suivant, indépendamment de l'année, du jour de l'année ou du jour de la semaine auquel correspondent les enregistrements. Ces 24 heures représentent un « jour ouvrable type » (CERTU, 1998).

Le produit de l'EMD reformaté dans un modèle de données séquentiel n'a pas besoin d'être représenté dans un formalisme classique type UML puisqu'il n'y a plus qu'un seul objet : l'individu. Il peut en revanche être représenté sous la forme d'un cube ( $c f$. Figure 3), dont les trois dimensions sont (i) l'individu, (ii) l'axe temporel, et (iii) les couches d'états qui recueillent les propriétés contenues dans l'objet déplacement et dans l'objet étape. Ce type de formalisme est semblable à celui proposé très tôt par Bonnafous et alii (1981) et plus récemment par Francis et Fuller (1996) puis par Francis et Pritchard (2000). La différence fondamentale repose dans le statut qui lui est assigné : ces auteurs en font un mode de représentation graphique alors que nous défendons l'idée qu'il s'agit d'un modèle conceptuel à même de guider l'ensemble des traitements et des interprétations réalisés sur les EMD. 


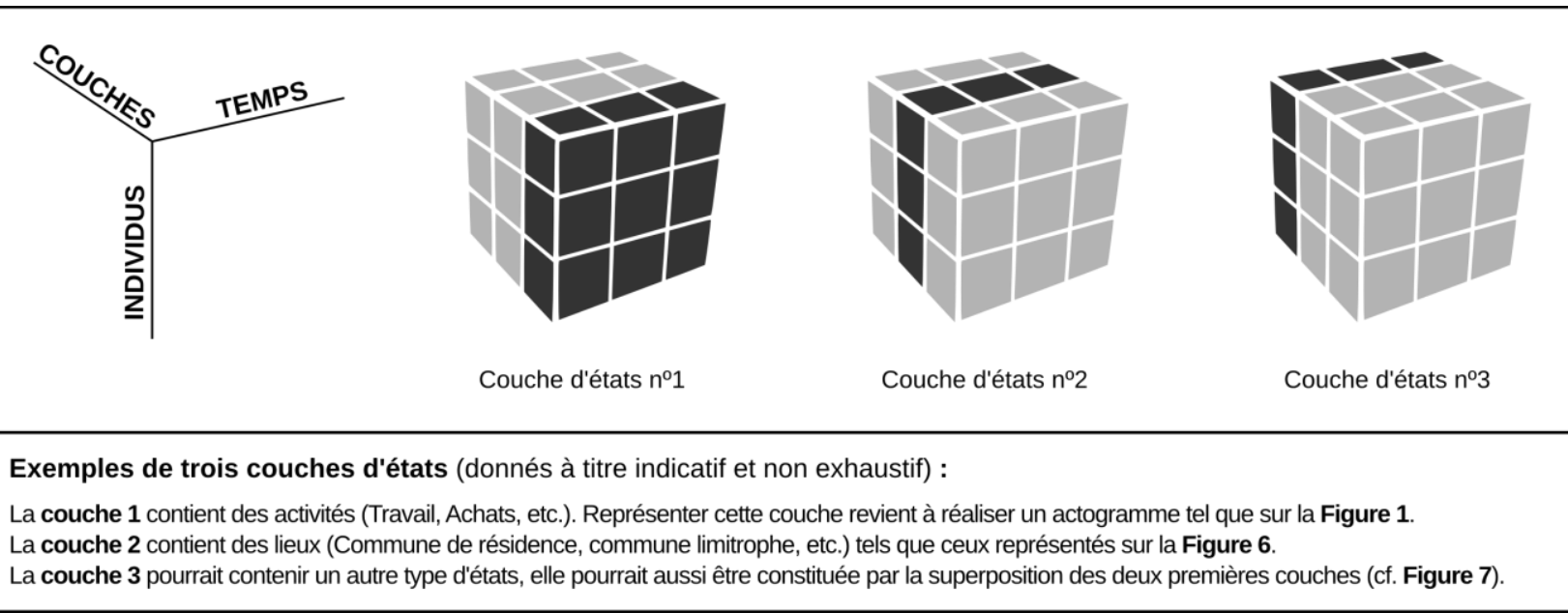

Réalisation : H.Commenges

\section{Caractéristiques et intérêt du modèle de données séquentiel}

En premier lieu, certaines caractéristiques du modèle séquentiel doivent être précisées, en particulier en termes de granularité. La granularité temporelle, c'est-à-dire la plus petite unité de l'axe temporel discret est nécessairement celle des données d'origine, à savoir une minute. Il est cependant possible de réaliser une agrégation à cinq minutes sans perte d'information conséquente. La granularité des états est évidemment celle des données d'origine. Cette granularité inclut la granularité spatiale (propriété des déplacement et des étapes) et la granularité des propriétés qualifiant les épisodes de mobilité (déplacement) et d'immobilité (activité).

Il est impossible de se départir de la division fondamentale du modèle de données originel entre mobilité et immobilité. Les propriétés qui les qualifient sont propres à chacun de ces deux états : les propriétés principales qualifiant les épisodes d'immobilité (les «stations » dans le vocabulaire de la time-geography) sont les activités, les propriétés principales qualifiant les épisodes de mobilité sont les modes. La caractérisation spatiale des états subit aussi cette division : les activités sont localisées dans des lieux fixes (l'état qui décrit la localisation est constant au cours de l'épisode), alors que les déplacements sont caractérisés par deux localisations, celle de l'origine et celle de la destination, l'entre-deux n'étant pas renseigné.

Le modèle de données historique introduit en effet une incompatibilité entre mobilité et activité. Plusieurs auteurs ont mis en avant que le déplacement n'est pas un entre-deux vide de sens, un temps mort entre deux activités (Orain, 1997 ; Mokhtarian et Salomon, 2001). Cette conception est pourtant inscrite dans les concepts et les méthodes de l'économie des transports qui considèrent le déplacement dans sa seule dimension de coût, mais elle est également intégrée dans le modèle de donnés de l'enquête à travers l'objet déplacement. Déplacement et activité se définissent en négatif et sont donc conceptuellement incompatibles : il n'y a pas d'activité durant le déplacement, le déplacement lui-même n'est pas une activité.

Le modèle de données séquentiel ne peut se départir de la division originelle entre mobilité et immobilité, en revanche il les met sur le même plan en les considérant indistinctement comme des états. Cette transformation est fondamentale car elle permet de mieux rendre compte de certains comportements de mobilité qui entrent mal dans le modèle de données classique : les déplacements qui constituent en euxmêmes l'activité, comme la promenade, ou les déplacements dont le motif ne désigne pas un lieu dans lequel se déroule une activité, comme l'accompagnement. Sans se départir totalement de la division mobilité/immobilité, il devient théoriquement possible d'introduire des données sur les activités réalisées 
pendant un déplacement (travailler dans un train par exemple), et également d'introduire la notion de multi-activité (travailler en accompagnant un enfant par exemple).

Ensuite, cette transformation des propriétés des objets déplacement et étape en états rend plus aisée l'intégration de nouveaux modes de recueil de données, en particulier de données GPS, qui commencent à s'imposer chez les organismes concernés (5). Dans le modèle de données séquentiel, les données GPS caractériseraient la localisation variable des états de mobilité, se rapprochant par là d'un modèle conceptuel spécialement conçu pour saisir des trajectoires (Spaccapietra et alii, 2008). Les trajectoires de la time-greography, trajectoires individuelles s'inscrivant dans un prisme spatio-temporel, sont difficiles à décrire et à représenter à partir du modèle de données historique, du fait que les stations (épisodes d'immobilité) n'en font pas partie. Le modèle de données séquentiel permet de repérer et d'analyser très facilement des trajectoires, sous la forme de patrons d'états (state patterns).

En outre, une lecture verticale des données séquentielles ( $c f$. Figure 1) amène facilement à des analyses de présence spatiale ou de rythmes urbains telles que celles réalisées par Banos et Thévenin (2004) et plus récemment par Bertrand et alii (2008). En effet, il suffit de prendre des séquences d'états représentant la localisation des individus et de les agréger pour chaque tranche temporelle pour obtenir une image de la présence des individus dans l'espace d'étude durant la journée.

Finalement, le modèle de données séquentiel offre la possibilité de créer des séquences multi-couches (Pollock, 2007), c'est-à-dire des séquences qui combinent plusieurs couches d'états caractérisant les épisodes mobiles et immobiles. Un exemple simple serait de combiner les états désignant les activités (domicile, travail, achats, etc.) avec les états désignant la localisation de ces états (par exemple, Paris, petite couronne, grande couronne).

Le modèle de données historique est bien adapté aux objectifs décrits dans la première section. Le modèle de données séquentiel ne s'y substitue en rien, il se justifie par sa complémentarité pour décrire les pratiques spatio-temporelles des individus. Dans le cadre d'une approche de ce type, il opère une grande simplification des schémas interprétatifs : il n'y a plus qu'un seul objet, l'individu, qui devient nécessairement l'objet d'intérêt. Dans ce modèle conceptuel, les analyses intègrent nécessairement la durée, à la fois des épisodes de mobilité et d'immobilité, alors que le modèle de données classique fait de la durée une propriété parmi d'autres de l'objet déplacement, et ne fait aucune place à la durée des épisodes d'immobilité. Enfin, le modèle de données séquentiel permet de reformuler partiellement le lien hérité du modèle de données classique : immobilité/activité et son corollaire mobilité/inactivité. En effet, les épisodes de mobilité et d'immobilité sont mis sur le même plan, à savoir des états qui caractérisent les individus tout au long de la journée.

\section{Complémentarité des deux modèles de données}

Dans le but de comparer les deux modèles de données, nous partons d'un exemple simple utilisant le modèle de données et le schéma interprétatif classiques avec deux variables fréquemment utilisées dans les études de mobilité et de transport. Ensuite, une analyse thématiquement comparable est réalisée avec le modèle de données séquentiel et les résultats issus de cette analyse sont confrontés aux premiers. La comparabilité est purement thématique, les traitements sont réalisés sur des propriétés différentes dans les deux cas mais, dans les deux cas, elles ont trait à la distance : distance parcourue d'abord, puis présence dans des espaces plus ou moins lointains.

\section{Analyse déplacement-centrée}

Le point de départ est l'analyse de la relation entre deux variables : le nombre de déplacements réalisés par individu et la distance totale parcourue. Il s'agit de variables analytiques caractérisant les individus par un résumé numérique sur les déplacements : un comptage et une somme. Ces deux variables sont ensuite utilisées pour réaliser une classification des individus autour de leurs médianes, respectivement 3 déplacements et $10,5 \mathrm{~km}$, pour aboutir à quatre types : les hypomobiles, les hypermobiles, les individus parcourant de longues distances concentrées sur peu de déplacements, et les individus parcourant de courtes distances distribuées sur de nombreux déplacements (6) (cf. Figure 4). 
Figure 4. Classification des individus selon le nombre de déplacements et la distance parcourue
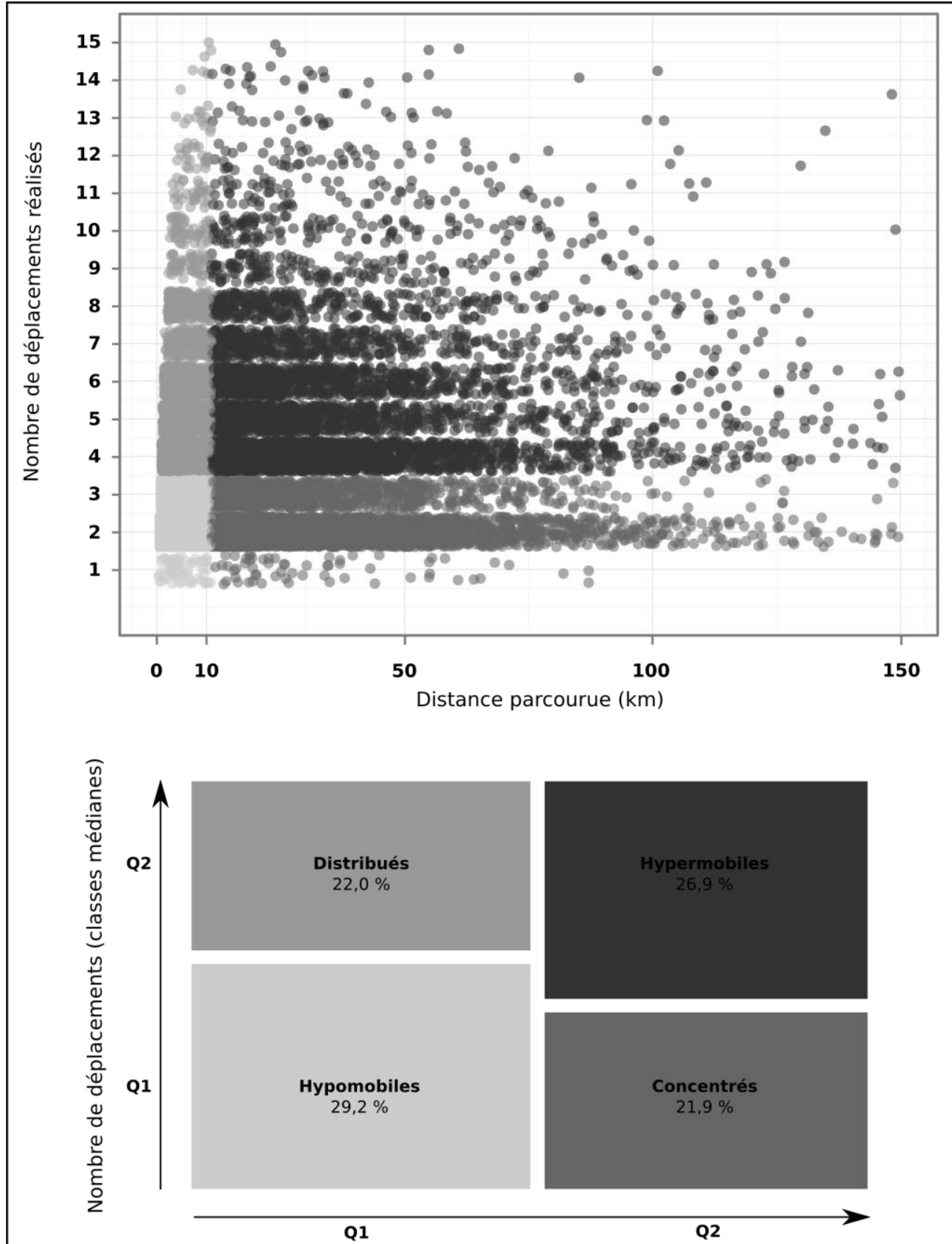

Distance parcourue (classes médianes)

\section{LECTURE DES GRAPHIQUES}

- Le nuage de points présente deux particularités : la variable Nombre de déplacements étant discrète, les points sont représentés sur une bande et non sur une ligne, et ils sont de plus partiellement transparents. Ces deux modifications du nuage de points classique ont pour but d'éviter la surcharge graphique (overplotting).

- Le graphique en mosaïque est une représentation du tableau de contingence. La surface des tesselles est proportionnelle à la fréquence, leur longueur sur les axes est proportionnelle à la fréquence marginale sur chacun des deux axes et représente ainsi les marges du tableau.

Source : Enquête Globale Transport 2001-2002 - Réalisation : H.Commenges 
Cette représentation est très schématique et n'a d'autre intérêt que de classer les individus en fonction de variables analytiques dérivées de l'objet déplacement. Sa seule raison d'être est d'amener à s'interroger sur les traitements de données sur la mobilité quotidienne et leur interprétation en posant une question simple : existe-t-il une relation entre la distance parcourue par les individus et le nombre de déplacements qu'ils réalisent? La partition binaire, autour des médianes respectives des deux variables, laisse entendre qu'il y aurait une relation positive entre les deux. Un examen plus précis, avec une partition plus fine de la variable distance et un test de comparaison de moyennes sur la variable Nombre de déplacements confirme ce résultat : plus la distance parcourue quotidiennement par les individus est grande, plus le nombre de déplacements qu'ils réalisent est important ( $c f$. Figure 5). C'est ce résultat qui sera discuté au regard de l'analyse réalisée dans le modèle de données séquentiel.

Figure 5. Relation entre distance parcourue et nombre de déplacements réalisés

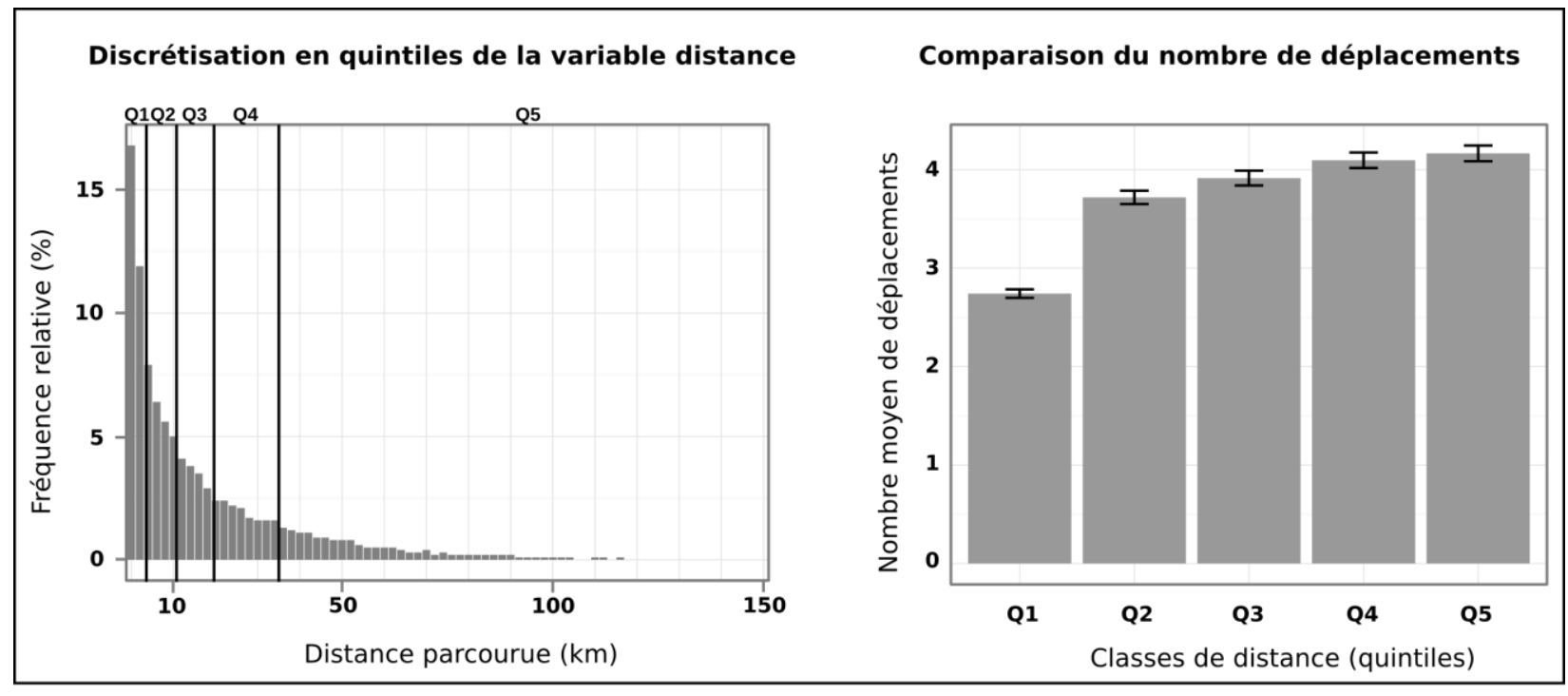

\section{DESCRIPTION DE L'ANALYSE}

- La distribution de la variable Distance est de type hyperbolique. Cette variable est discrétisée en quintiles et utilisée comme variable indépendante.

- La distribution de la variable "Nombre de déplacements" se rapproche également d'une hyperbole. Elle présente cependant un fort déséquilibre entre les nombres de déplacements pairs et impairs, dû au fait que la forme dominante des sorties est binaire (aller/retour). Cette variable est la variable dépendante de l'analyse.

- Les prérequis pour l'utilisation des méthodes paramétriques d'analyse de la variance et comparaison de moyennes ne sont pas respectés. Le test de Wilcoxon, non paramétrique, est donc utilisé.

- Tous les calculs et les tests prennent en compte les effets de grappe dus au fait que ce sont les ménages et non les individus qui constituent les unités de tirage (PSU - Primary Sampling Units).

- Toutes les moyennes sont significativement différentes les unes des autres, à l'exception des deux derniers quintiles Q4 et Q5.

Source : Enquête Globale Transport 2001-2002 - Réalisation : H.Commenges 


\section{Analyse séquentielle}

Le modèle de données séquentiel permet de s'intéresser non plus seulement aux déplacements des individus mais à leurs pratiques spatiales, plus précisément à la fréquentation de durée variable des espaces. L'analyse est menée en quatre temps, chacun détaillé dans une figure dédiée.

1. Créer un zonage de distance à partir d'une définition relative et individu-centrée des distances, en forme de couronnes. Comme les coquilles de Moles et Rohmer (1978) ou plus récemment les couronnes de destinations touristiques (Berroir et alii, 2011), chaque individu est doté d'un zonage qui lui est propre, constitué de couronnes d'éloignement autour de sa commune de résidence. Ces couronnes comportent quatre modalités : commune de résidence, communes limitrophes, communes proches et communes lointaines ( $c f$. Figure 6).

2. Classer les individus sur un critère de variabilité temporelle de la présence spatiale. Il ne s'agit pas de les classer sur le critère de la distance plus ou moins importante qu'ils parcourent mais sur le critère des espaces plus ou moins lointains qu'ils fréquentent. Le préalable est de réunir deux couches d'états caractérisant les individus : la première couche distingue les épisodes au domicile et hors du domicile (deux modalités); la seconde couche distingue les épisodes passés dans les différentes couronnes de distance autour du domicile (quatre modalités). La superposition des deux aboutit à un ensemble de séquences à cinq modalités : domicile, commune de résidence (hors domicile), commune limitrophe, commune proche, commune lointaine. La classification est ensuite réalisée sur le temps passé dans chacun de ces états ( $c f$. Figure 7).

3. Récupérer les groupes d'individus créés par la classification des séquences spatialisées et les projeter sur le graphique mettant en relation nombre de déplacements et couronnes de distance fréquentées. L'analyse du graphique obtenu ( $c f$. Figure 8 ) montre un patron radial où les individus qui fréquentent les espaces les plus proches sont aussi ceux qui réalisent le plus grand nombre de déplacements.

4. Faire une comparaison du nombre moyen de déplacements réalisés en fonction des groupes créés par l'analyse de présence spatiale, par un test de comparaison de moyennes identique à celui réalisé dans l'analyse déplacement-centrée ( $c f$. Figure 9). Ce test confirme le résultat visuel obtenu dans la Figure 8, il y a une relation négative significative entre les deux variables : plus les individus sont présents dans des espaces lointains, moins ils réalisent de déplacements.

\section{Figure 6. Zonage en couronnes de distance}

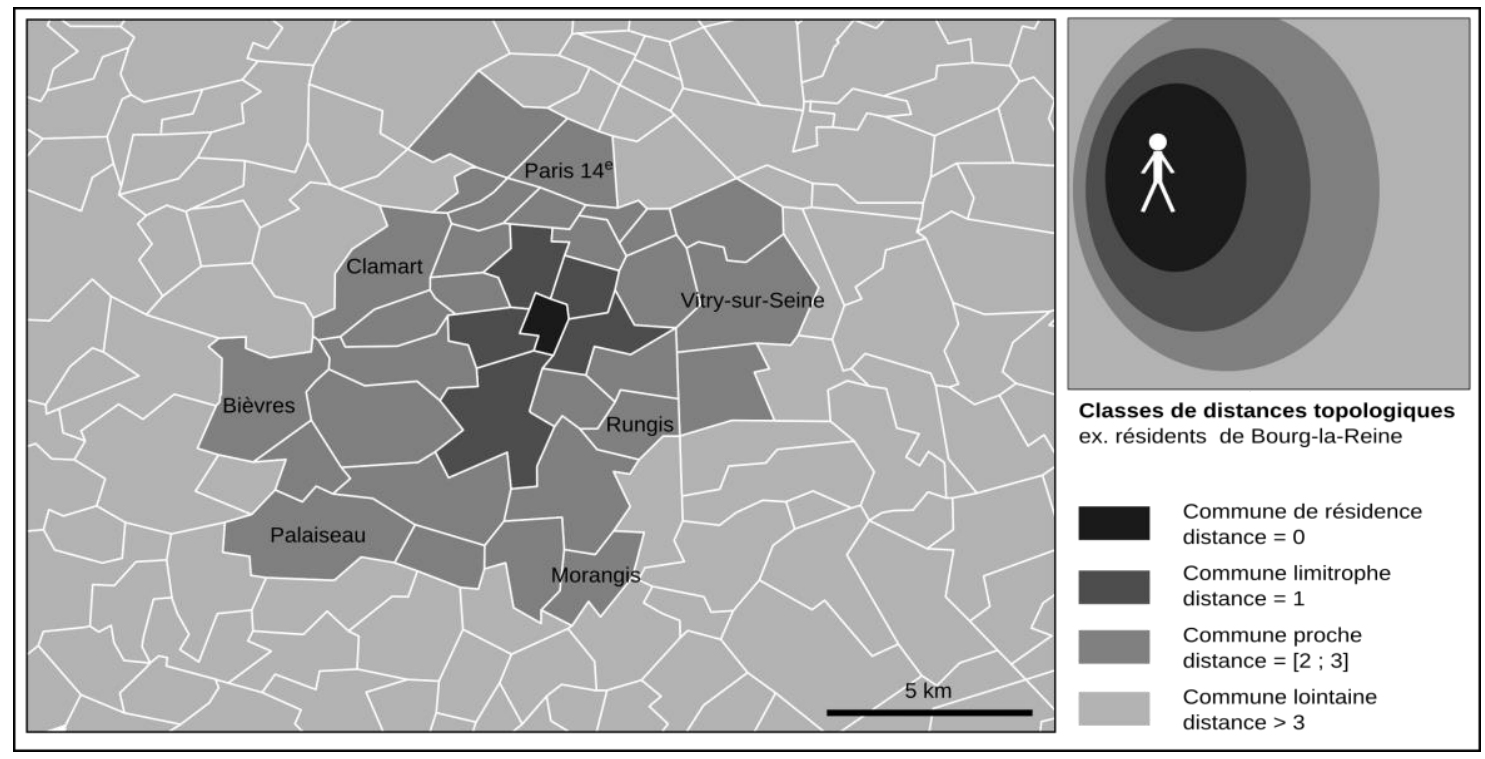

Source : Enquête IAU-IdF, Communes simplifiées - Réalisation : H.Commenges 
Figure 7. Classification des séquences spatialisées (échantillon de 20 individus)

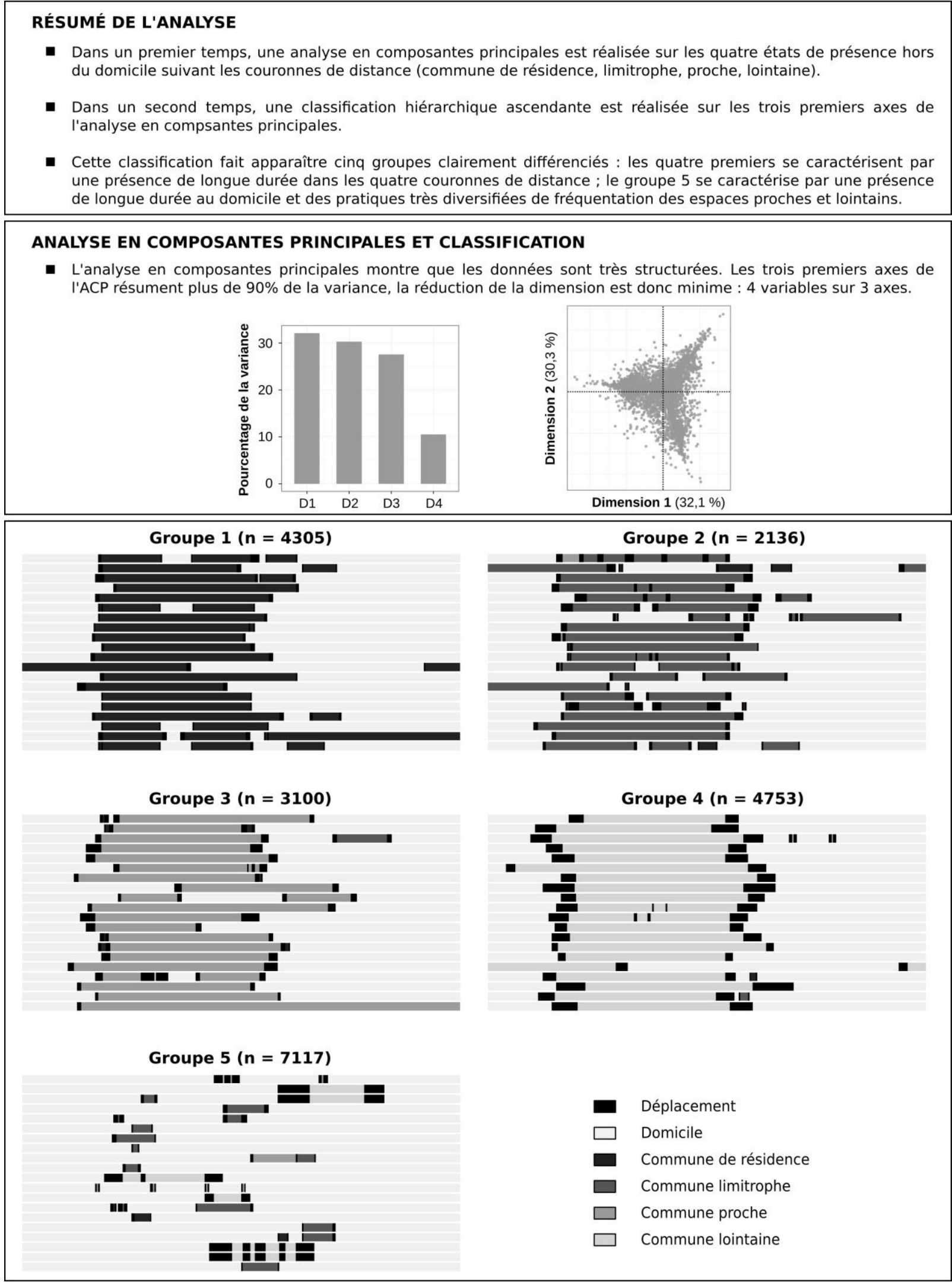

Source : Enquête Globale Transport 2001-2002 - Réalisation : H.Commenges 
Figure 8. Projection des groupes selon nombre de déplacements et distance parcourue

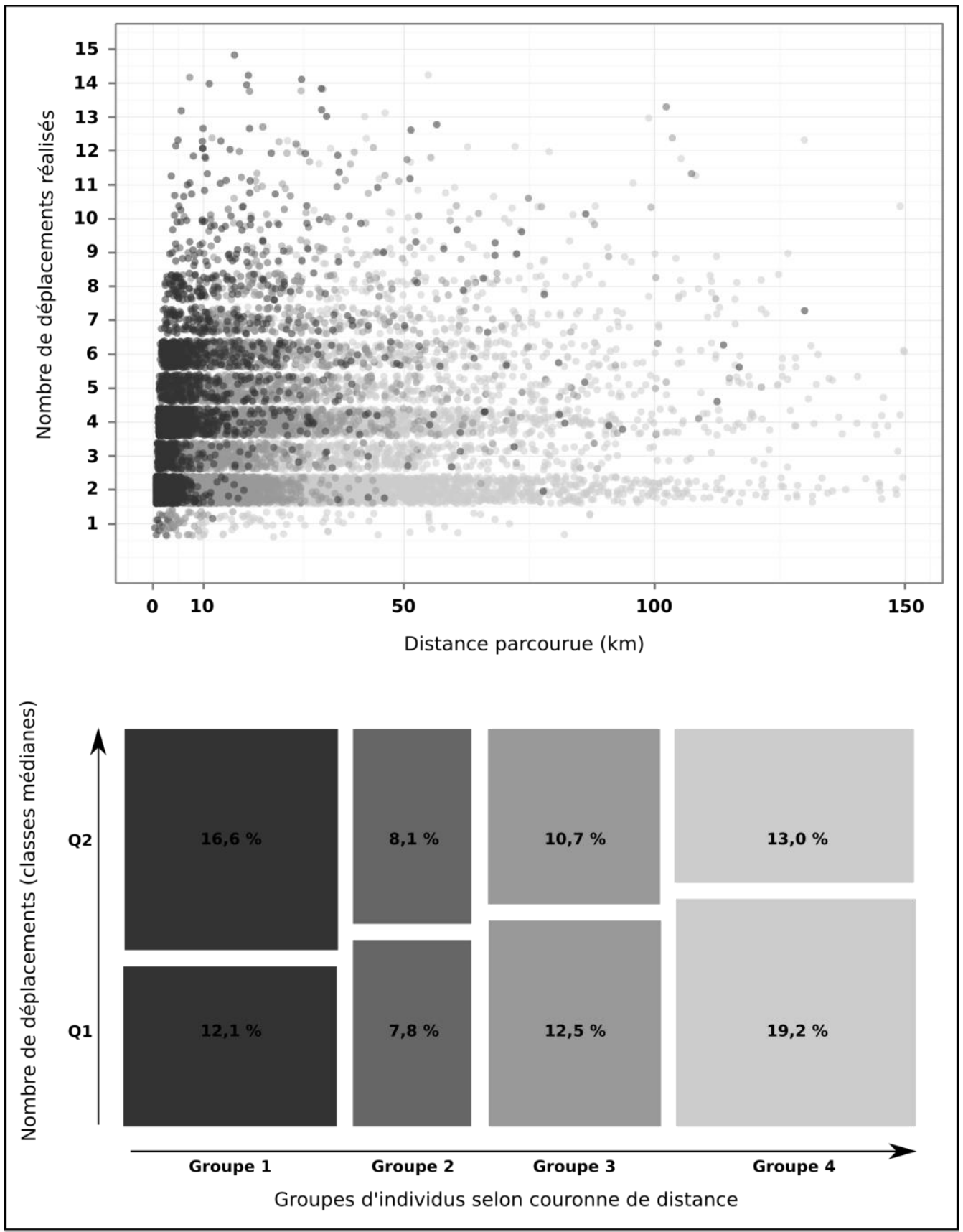

\section{LECTURE DES GRAPHIQUES}

- L'analyse est réalisée sur les quatre premiers groupes issus de la classification précédente. Le groupe 5 présente une grande hétérogénéité vis-à-vis des couronnes d'éloignement fréquentées. Il est exclu de l'analyse.

- Le nuage de points présente deux particularités : la variable Nombre de déplacements étant discrète, les points sont représentés sur une bande et non sur une ligne, et ils sont de plus partiellement transparents. Ces deux modifications du nuage de points classique ont pour but d'éviter la surcharge graphique (overplotting).

- Le graphique en mosaïque est une représentation du tableau de contingence. La surface des tesselles est proportionnelle à la fréquence, leur longueur sur les axes est proportionnelle à la fréquence marginale sur chacun des deux axes et représente ainsi les marges du tableau.

Source : Enquête Globale Transport 2001-2002 - Réalisation : H.Commenges 
Figure 9. Relation entre groupes de fréquentation spatiale et nombre de déplacements réalisés

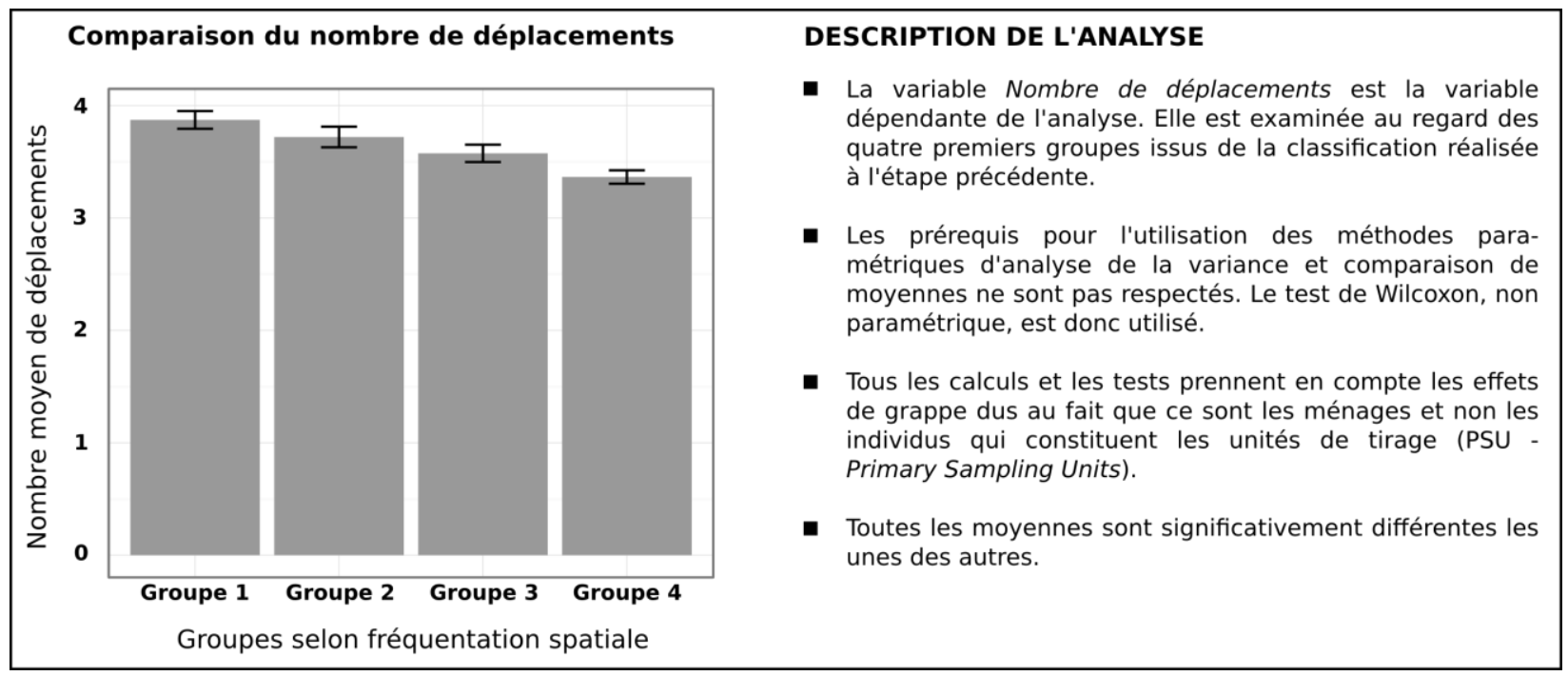

Source : Enquête Globale Transport 2001-2002 - Réalisation : H.Commenges

Ainsi, cette seconde analyse mène à la conclusion que plus les individus fréquentent des espaces proches de leur domicile, plus ils réalisent de déplacements, et son corollaire, plus ils fréquentent des espaces lointains, moins ils réalisent de déplacements. Ce résultat est très différent de celui obtenu en ne prenant en compte que les épisodes de mobilité. En effet, l'analyse déplacement-centrée se focalise sur les états mobiles des individus et ne tient pas compte de tout ce qui se passe, « en creux », entre les déplacements, à savoir la présence spatio-temporelle des individus.

À la différence de l'approche classique qui considère que la mobilité s'exprime en termes de déplacements, le modèle séquentiel amène à considérer la mobilité comme la variabilité temporelle de la présence spatiale, dont les épisodes d'immobilité font partie au même titre que les épisodes de mobilité. Cette façon de concevoir la mobilité ramène à des considérations déjà anciennes dans le cadre de la timegeography : "Why should geography be unduly crippled by not facing up the fact that much interaction is in the form of stationary activity which implies movement through time inside stations such as buildings and premises » (Carlstein et alii, 1978, p. 12). Carlstein et alii ajoutent plus loin : «the spatial-areal arrangement of phenomena and processes and their arrangement in time are part of the same thing [...] To cull out mobile activities from the context of the stationary activities with which they interconnect is a dangerous form of sectorization ». La confrontation des deux approches réalisée dans cet article illustre le risque pointé par ces auteurs et montre l'intérêt d'une approche complémentaire.

\section{Conclusion}

Le premier objectif de ce travail était de montrer comment un modèle conceptuel de données peut ménager un chemin qui canalise les questionnements, les traitements et les interprétations. Le modèle de données des EMD est déplacement-centré depuis la naissance de ces enquêtes durant les années 1940 aux États-Unis (Weiner, 1997). Il a conservé ces caractéristiques jusqu'à aujourd'hui pour les besoins opérationnels des administrations et des gestionnaires d'infrastructures et de services de transport. Dans la lignée de la time-geography, la recherche en mobilité et transports s'est enrichie dès les années 1970 d'approches par les modes de vie et d'approches activités-centrées. Mais ces recherches ne se sont pas suffisamment imposées en France pour justifier la mise en place d'un dispositif d'enquête répondant mieux à leurs besoins.

L'objectif de cet article était également de montrer qu'il est possible de reconstruire un modèle de données séquentiel individu-centré à partir du modèle de données traditionnel. Il s'agissait également de défendre 
l'idée que cette reformulation est plus qu'un mode de représentation graphique mais bien une façon de réorganiser l'information contenue dans une EMD autour d'un seul objet, l'individu, assorti d'un ensemble de propriétés temporelles. Enfin, une analyse simple réalisée sur le modèle de données séquentiel a montré le caractère partiel des analyses strictement déplacement-centrées (Carlstein et alii, 1978). Le modèle de données séquentiel amène facilement à une analyse en terme de variabilité temporelle de la présence spatiale. Celle-ci ne se substitue pas à la première, mais elle apporte un éclairage différent et complémentaire, ce qui plaide en faveur d'une multiplication des approches. Le modèle de données séquentiel permet enfin de penser, de traiter et d'interpréter la mobilité en tant que trajectoires individuelles ou en tant que rythmes urbains, approches qui auraient certainement apporté un éclairage différent sur notre exemple.

\section{Notes}

(1) C'est ce terme qui sera utilisé ici, à la fois parce que c'est le nom du dispositif normalisé par les services ministériels français (SETRA, puis CETUR, puis CERTU), et parce que c'est la traduction littérale du terme anglosaxon désignant le dispositif d'enquête originel : Household Travel Survey.

(2) La terminologie utilisée ici, qui distingue variables absolues et variables contextuelles, est tirée de la grille de lecture de Lazarsfeld et Menzel (1961).

(3) Et dans l'objet étape, mais cet objet ne sert finalement qu'à préciser l'objet déplacement.

(4) Toutes les représentations graphiques de séquences et les mesures réalisées sur ces séquences sont réalisées avec la bibliothèque TraMineR du logiciel $\mathrm{R}$ (Gabadinho et alii, 2011).

(5) Le CERTU, les partenaires de l'EGT, l'IFSTTAR, l'INSERM : de plus en plus de producteurs d'études et de recherches intègrent ou réfléchissent à l'intégration de données GPS dans les enquêtes de mobilité. Voir l'état de la question dressé par Nguyen-Luong (2012).

(6) Tous les traitements sont réalisés sur l'échantillon de l'EGT 2001-2002, à savoir près de 21000 individus. Environ 3000 individus sont exclus de l'échantillon initial : les individus immobiles, les individus dont certains déplacements ne sont pas renseignés ou mal renseignés, les individus dont les valeurs pour ces deux variables sont des outliers, à savoir ceux qui réalisent plus de 15 déplacements quotidiens et ceux qui parcourent des distances supérieures à $150 \mathrm{~km}$. Les calculs de distance sont réalisés sur l'objet étape en suivant la même méthode que celle de l'étude sur les distances réalisée par l'IAU-IdF (Courel, 2008). Toutes les mesures et les tests calculés prennent en compte les effets de grappe grâce aux fonctions développées par Lumley (2004) dans la bibliothèque survey du logiciel R.

\section{Bibliographie}

Abbott A., 1995, "Sequence analysis: new methods for old ideas", Annual Review of Sociology 21, pp. 93113.

Abbott A., Hysrcha A., 1990, "Measuring resemblance in sequence data: an optimal matching analysis of musicians' careers", American Journal of Sociology, 96, pp. 144-185.

As D., 1978, "Studies of time-use: problems and prospects", Acta Sociologica, 21, pp. 125-141.

Banos A., Thévenin Th., 2004, « Révéler les rythmes urbains par la carte animée », Revue internationale de géomatique, 15, pp. 11-32.

Bernard S., Debrincat L., Siméon Th., 2005, «Les déplacements en transports collectifs en Île-deFrance », Les Cahiers de l'EGT, vol.5, DREIF

Berroir S., Cattan N., Commenges H., Decroly J.-M., Fleury A., Guérois M., 2011, « Entre banalité et exotisme, le panel individuel des destinations touristiques », Mondes du Tourisme, Hors-série, pp. 50-62.

Bertrand F., Couderc C., Ladegaillerie E., 2008, « La dynamique des activités en Île-de-France », Les Cahiers de l'EGT, vol.10, DREIF 
Blossfeld H.-P., Rohwer G., 2002, Techniques of event history modeling: new approaches to causal analysis, Mahwah : Lawrence Erlbaum

Bonnafous A., Patier-Marque D., Plassard F., 1981, Mobilité et vie quotidienne, Lyon : Presses universitaires de Lyon

Brzinsky-Fay C., Kohler U., Luniak M., 2006, "Sequence analysis with Stata”, The Stata Journal, 6(4), pp. $435-460$

Carlstein T., Parkes D., Thrift N., 1978, Human activity and time-geography, New York : Wiley and Sons

CERTU, 1998, L'enquête ménages déplacements méthode standard. Notes méthodologiques et annexes, Lyon : CERTU

Chapin S. F., 1978, "Human time allocation in the city", in: Carlstein T., Parkes D., Thrift N. (dir.), Human activity and time-geography, New York: Wiley and Sons, pp. 13-26

Chardonnel S., Du Mouza C., Fauvet M.-C., Josselin D., Rigaux Ph., 2004, « Patrons de mobilité : proposition de définition, de méthode de représentation et d'interrogation », $7^{\text {ème }}$ Journées Cassini, Grenoble, 2-4 juin.

Chatzis K., 2009, « De l'importation des savoirs américains à la création d'une expertise nationale : la modélisation des déplacements urbains en France, 1950-1975 », in: Flonneau M., Guigueno V. (dir.), De l'histoire des transports à l'histoire de la mobilité ?, Rennes : Presses Universitaires de Rennes, pp. 159-169.

Commenges H., 2013, L'invention de la mobilité quotidienne. Aspects performatifs des instruments de la socio-économie des transports, Thèse de doctorat, Université Paris Diderot-Paris 7.

Courel J., 2008, 170 millions de kilomètres par jour. Les distances parcourues par les Franciliens quotidiennement, Paris : IAU-IdF

Courel J., Méyère A., Nguyen-Luong D., 2005a, « L'impact des modes de vie sur les déplacements », Les Cahiers de l'EGT, vol.4, DREIF

Courel J., Méyère A., Nguyen-Luong D., 2005b, «Répartition géographique des déplacements : une nouvelle approche », Les Cahiers de l'EGT, vol.3, DREIF

Desrosières A., Thévenot L., 1979, « Les mots et les chiffres : les nomenclatures socio-professionnelles », Economie et statistique, 110, pp. 49-65.

Desrosières A., 1989, «Comment faire des choses qui tiennent : histoire sociale et statistique », Histoire et Mesure, 4(3), pp. 225-242.

Didier E., 2009, En quoi consiste l'Amérique : les statistiques, le New Deal et la démocratie, Paris : La Découverte

Dreyfus J., 2005, «Le profil des déplacements journaliers en transports en commun et voiture particulière », Les Cahiers de l'EGT, vol.2, DREIF

Dupuy G., 1975, Une technique de planification au service de l'automobile. Les modèles de trafic urbain, Paris : Ministère de l'équipement

Facq B., 2006, Les fondements statistiques de la science française des déplacements urbains, Mémoire de master, Lyon : Institut de d'Études Politiques de Lyon

Francis B., Fuller M., 1996, "Visualisation of event histories", Journal of the Royal Statistical Society, Series A, 159, pp. 301-108.

Francis B., Pritchard J., 2000, « Bertin, Lexis and the graphical representation of event histories », Cybergeo : European Journal of Geography, [En ligne], Dossiers, Colloque « 30 ans de sémiologie graphique », document 152, mis en ligne le 17 novembre 2000, consulté le 23 mars 2014. URL : http://cybergeo.revues.org/506 ; DOI : 10.4000/cybergeo.506 
Gabadinho A., Ritschard G., Studer M., Mueller N. S., 2009, Mining sequence data in R with the TraMineR package. A user's guide, University of Geneva

Gabadinho A., Ritschard G., Mueller N.S., Studer M., 2011, “Analyzing and Visualizing State Sequences in R with TraMineR”, Journal of Statistical Software, 40(4), pp. 1-37.

Haumont A., Lamberti A., Viet-Depaule N., Wintersdorff F., 1977, La mobilité des citadins. Mobilité et modes de vie, Paris : Institut de Sociologie Urbaine

Haumont A., Lamberti A., Viet-Depaule N., Wintersdorff F., 1978. La mobilité des citadins. Mobilité et espaces urbains, Paris : Institut de Sociologie Urbaine

Ihl O., Kaluszynski M., Pollet G., 2003, Les sciences de gouvernement, Paris : Economica

Joh C.-H., Arentze T., Hofman F., Timmermans H., 2002, “Activity pattern similarity: a multidimensional sequence alignment method", Transportation Research Part B, 36, pp. 385-403.

Lascoumes P., Le Galès P., 2004, Gouverner par les instruments, Paris : Presses de Sciences Po

Lazarsfeld P. F., Menzel H., 1961, "On the relation between individual and collective properties", in: Etzioni A., Lehman E. W. (eds.), Complex organizations : a sociological reader, New York: Holt, Rinehart and Winston, pp. 508-521

Lumley Th., 2004, “Analysis of complex survey samples”, Journal of Statistical Software, 9, pp. 1-19.

Mokhtarian P., Salomon I., 2001, "How derived is the demand for travel? Some conceptual and measurement considerations", Transportation Research Part A, 35, 8, pp. 695-719.

Ministère des Travaux Publics, 1918, Routes nationales. Recensement de la circulation en 1913, Paris : Ministère des travaux publics

Moles A., Rohmer E., 1978, Psychologie de l'espace, Paris : Casterman

Nguyen-Luong D., 2012, Faisabilité d'une enquête globale transport intégrale par association d'un GPS, d'un SIG et d'un système expert en Île-de-France, Paris : IAU-IdF

Orain H., 1997, « Du côté des trajets. Types de mobilités quotidiennes », in: Juan J. (dir.), Les sentiers du quotidien : rigidité, fluidité des espaces sociaux et trajets routiniers en ville, Paris : L'Harmattan, pp. 97-119

Parent Ch., Spaccapietra S., Zimányi E., Donini P., Plazanet C., Vangenot Ch., Rognon N., Crausaz P.-A., 1997, «MADS, modèle conceptuel spatio-temporel », Revue Internationale de Géomatique, 7, pp. 317-352.

Perec G., 1978, La vie mode d'emploi, Paris : Hachette

Pollock G., 2007, "Holistic trajectories: a study of combined employment, housing and family careers by using multiple-sequence analysis", Journal of the Royal Statistical Society : Series A, 170, pp. 167-183.

Raux Ch., Andan O., Bonnel P., 1988, Les analyses des comportements de mobilité individuelle quotidienne : une synthèse bibliographique, Lyon : Laboratoire d'Économie des Transports

SERC, 1964, Études de transports urbains. Analyse des méthodes américaines, SERC

SERC, BRC de Rennes, 1964, Étude de circulation : Rennes, SERC

SETRA, 1975, Dossier pilote concernant la réalisation des enquêtes ménages, Bagneux : SETRA

Salais R., Reynaud B., Baverez N., 1986, L'invention du chômage. Histoire et transformations d'une catégorie en France des années 1890 aux années 1980, Paris : Presses Universitaires de France

Schor P., 2001, Compter et classer : histoire des catégories de la population dans le recensement américain, 1790-1940, Thèse de doctorat, EHESS 
Spaccapietra S., Parent Ch., Damiani M. L., Macedo (de) J.-A., Porto F., Vangenot Ch., 2008, “A conceptual view on trajectories", Data and Knowledge Engineering, 65, pp. 126-146.

Taris T. W., 2000, A primer in longitudinal data analysis, London : SAGE Publications

Thévenin Th., Chardonnel S., Cochey E., 2007, « Explorer les temporalités urbaines de l'agglomération de Dijon : une approche activité-centrée de l'Enquête Ménage Déplacement », Espace Populations Sociétés, pp. 2-3.

Weiner E., 1997, Urban transportation planning in the United States: an historical overview, Westport : Praeger Publishers 